\section{To Be, Notch to Be}

\author{
Moumita De ${ }^{1} \quad$ Rakesh Dawar $^{1} \quad$ Maneesh Singhal ${ }^{1}$ \\ ${ }^{1}$ Department of Plastic, Reconstructive and Burns Surgery, All India \\ Institute of Medical Sciences, New Delhi, India
}

Indian J Plast Surg 2020;53:154-156

The use of full-thickness grafts (FTG) to resurface small to medium-sized wounds are popular in plastic surgical practice due to good aesthetic outcome and primary closure of the donor site. However, complication like wound dehiscence of the donor site is a limitation, especially in cases of a large area of harvest, where the wound is tightly closed. Conventionally, FTG is taken in a biconvex shape to facilitate the closure. In our clinical practice, we observed that the central portion of the wound bears maximum tension; hence, it is more prone to dehiscence. After seeing a few cases of dehiscence over the years, we propose a simple modification in the design of graft that both facilitates closure and prevents dehiscence. We call this "notch design" as its shape looks like a notch on smartphone display.

The groin is the preferred donor site for most FTGs. ${ }^{1}$ Laxity of skin and presence of natural groin crease augments harvest of large size grafts and aids primary closure of donor site. As large as $25 \times 20 \mathrm{~cm}^{2 \mathrm{FT}} \mathrm{G}$ has been described, where surgeons achieved primary closure, ${ }^{2}$ but a study by Kim et al mentioned donor wound dehiscence complication rate up to $6 \%$ for maximum dimensions of $8 \mathrm{~cm} \times 13 \mathrm{~cm} .{ }^{3}$

In our cases, we have taken grafts of width ranging from $2 \mathrm{~cm}$ to $9 \mathrm{~cm}$ and length from $5 \mathrm{~cm}$ to $13 \mathrm{~cm}$. These are most commonly used in patients of syndactyly for resurfacing post release. We have used small pieces of full grafts as per need of defect. In traditional described elliptical design, the ratio of width to length is $1: 3$, which is reliable enough to achieve the primary closure. Observing the nature of wound dehiscence in the center ( $\boldsymbol{-}$ Fig. 1), we concluded that most common site of dehiscence is the central part of the wound, due

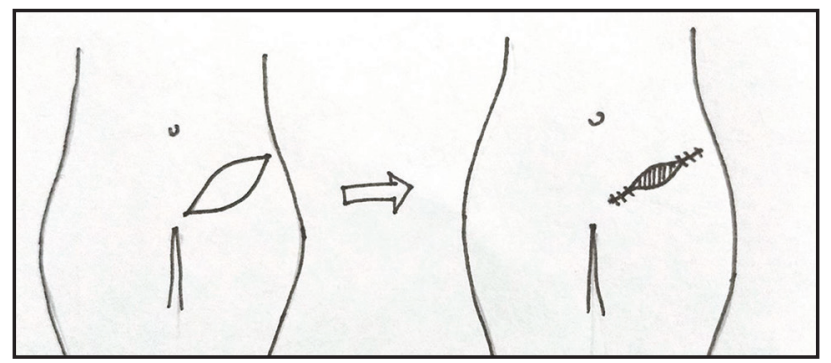

Fig. 1 Elliptical design with dehiscence at the center.
Suvashis Dash ${ }^{1}$ Ashish Bichpuriya ${ }^{1}$

Address for correspondence Maneesh Singhal, MS, MCh, FACS, FRCS (Glasgow), Department of Plastic, Reconstructive and Burns Surgery, All India Institute of Medical Sciences, Room 5014C, Teaching Block, New Delhi 110029, India (e-mail: drmaneesh@gmail.com).

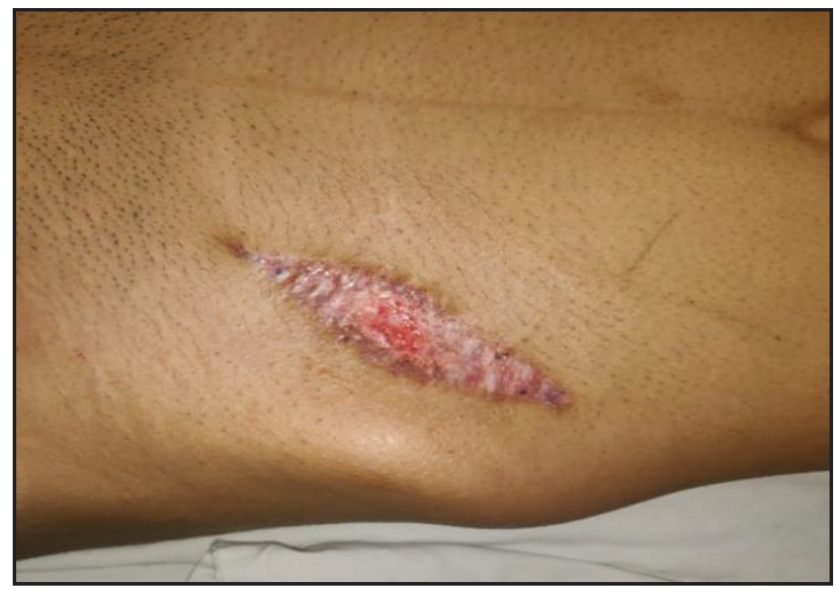

Fig. 2 Wound with dehiscence at the center.

to maximum width, in turn, it had to withstand maximum tension (-Fig. 2).

\section{Biogeometry}

The idea was to reduce tension at the center of the wound, by reducing width in the center without decreasing substantial amount of graft area ( - Fig. 3). Maximum width of the wound $(\mathrm{MN})$ is marked by "X" ( - Fig. 4). The length of graft (AB) was divided into thirds ( $A C^{\prime}, C^{\prime} C$," and $\left.C^{\prime \prime} B\right)$. At the points C' and ' $C^{\prime \prime}$ two lines perpendiculars $A B$ were drawn through the whole width, which joins the margins of graft at D, D', E, and E'. In the proposed design, we decreased the width $\mathrm{X}$ in the center by $25 \%(\mathrm{Y})$. This was done by adding notch from points $\mathrm{D}$ and $\mathrm{D}$ ', down to the central point of line (M' point). This way, effective width at center is $\mathrm{X}-\mathrm{Y}$ (represented by line $\mathrm{M}^{\prime} \mathrm{N}$ ), while very little skin marked by shaded area decreased (-Fig. 5). The lines DE and D'E' become the widest part of the graft.

After modifying the design of graft harvest, we have not encountered any wound complications for the past 6 months (38 patients, 40 FTGs, 2 being bilateral). A notch design on the smart phone display may be irritating, but this notch on the graft design is a noteworthy addition to our skill display (-Figs. 6-9). published online

March 26, 2020
DOI https://doi.org/

10.1055/s-0040-1708223

ISSN 0970-0358. (c) 2020 Association of Plastic

Surgeons of India
License terms

$\circledast(1) \Theta \circledast$ 


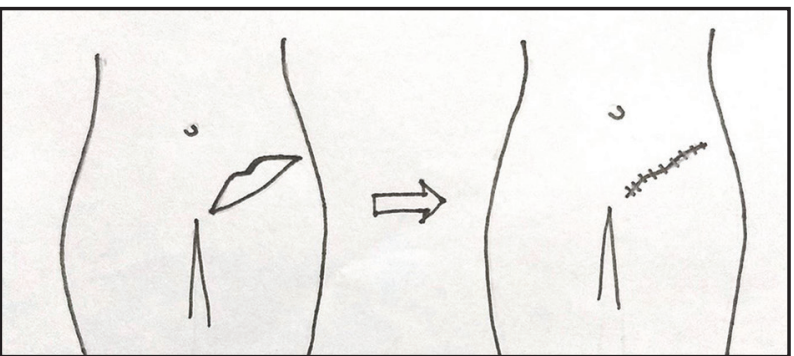

Fig. 3 Proposed “notch" design.

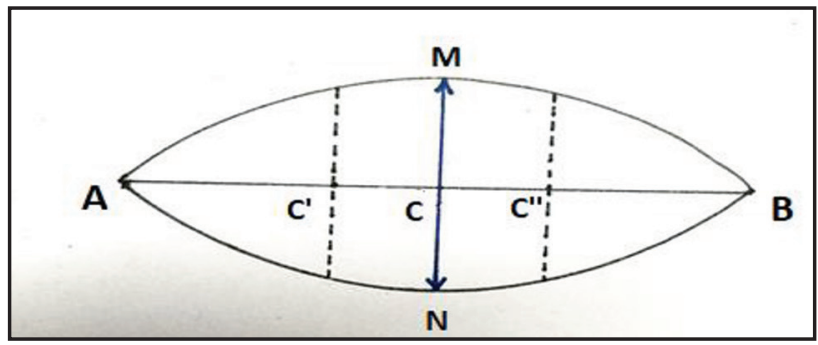

Fig. 4 Standard elliptical design (vide text).

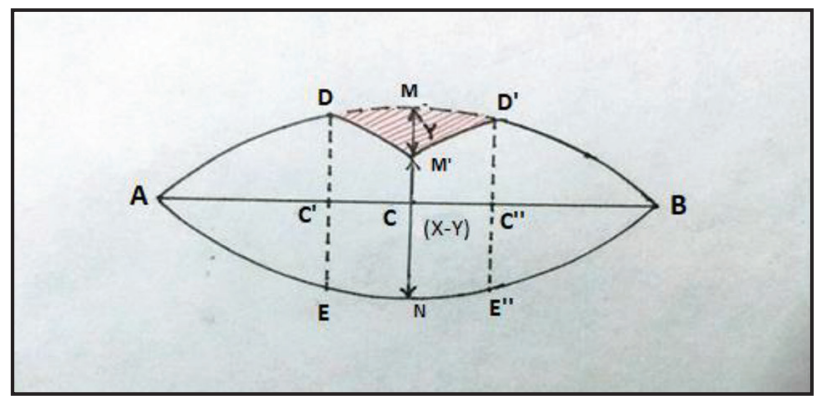

Fig. 5 Biogeometry of notch design (vide text).

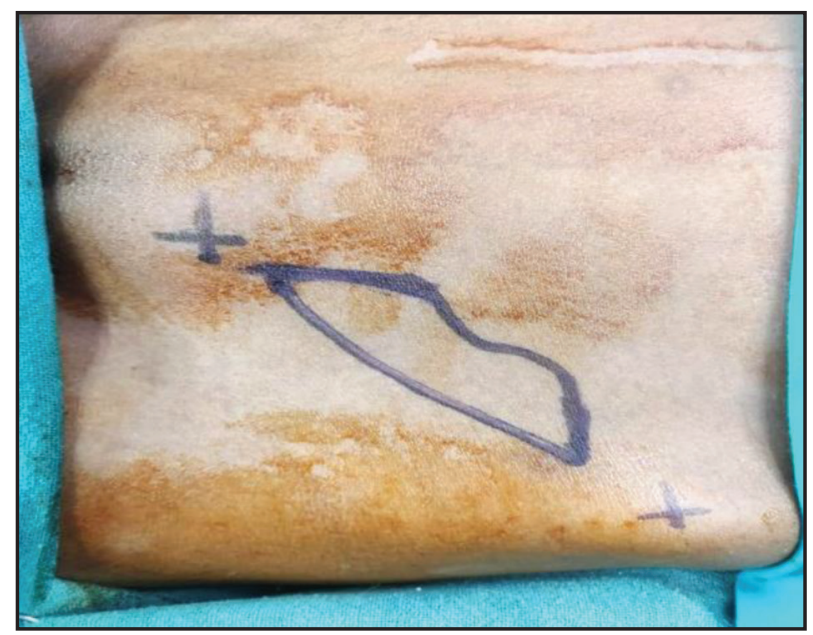

Fig. 6 Markings on a patient.

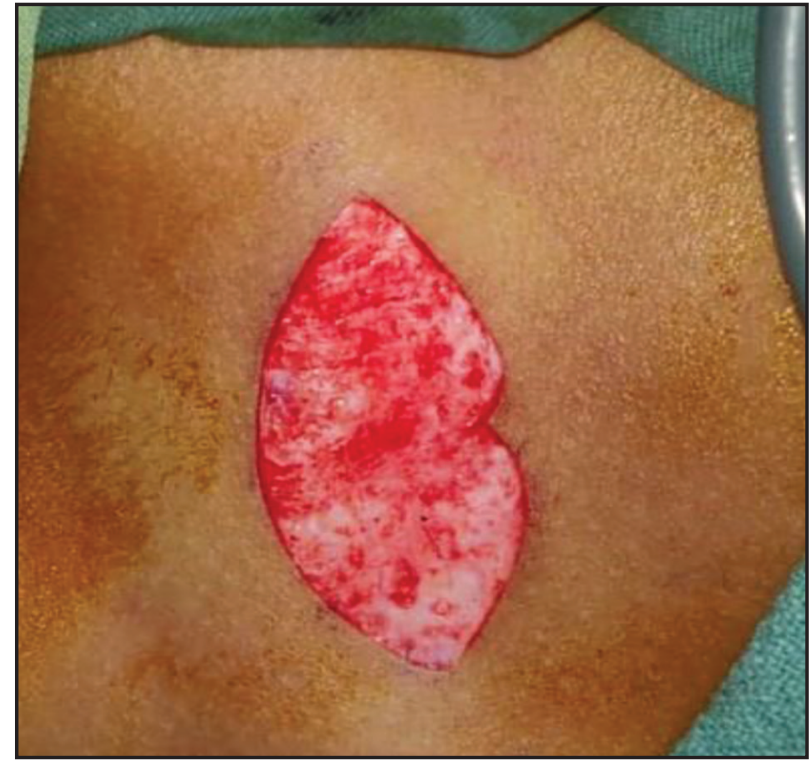

Fig. 7 Graft harvest with new design.

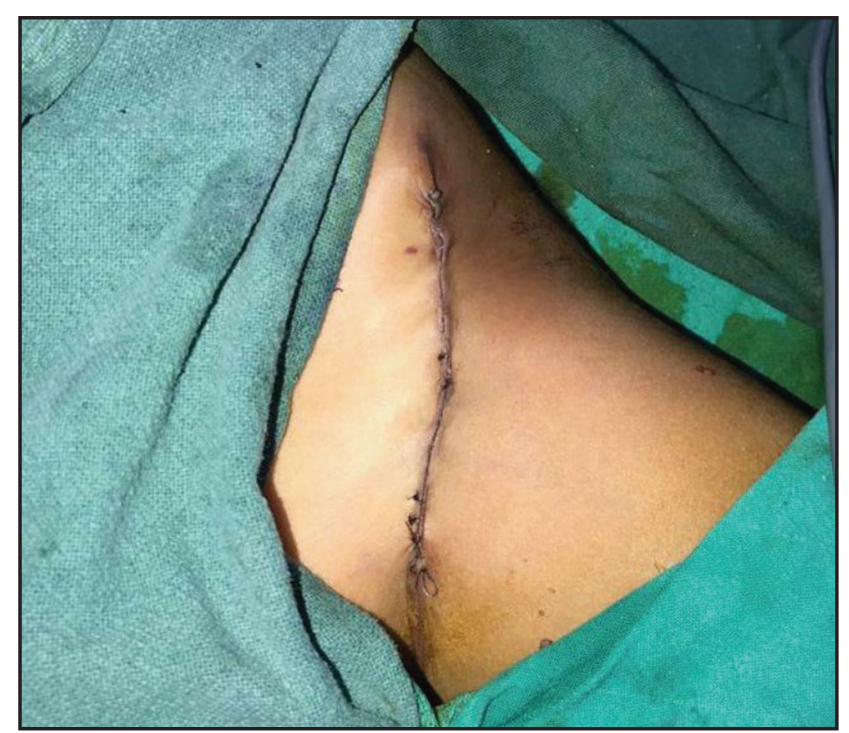

Fig. 8 Immediate postoperative picture of wound closure by our method. 


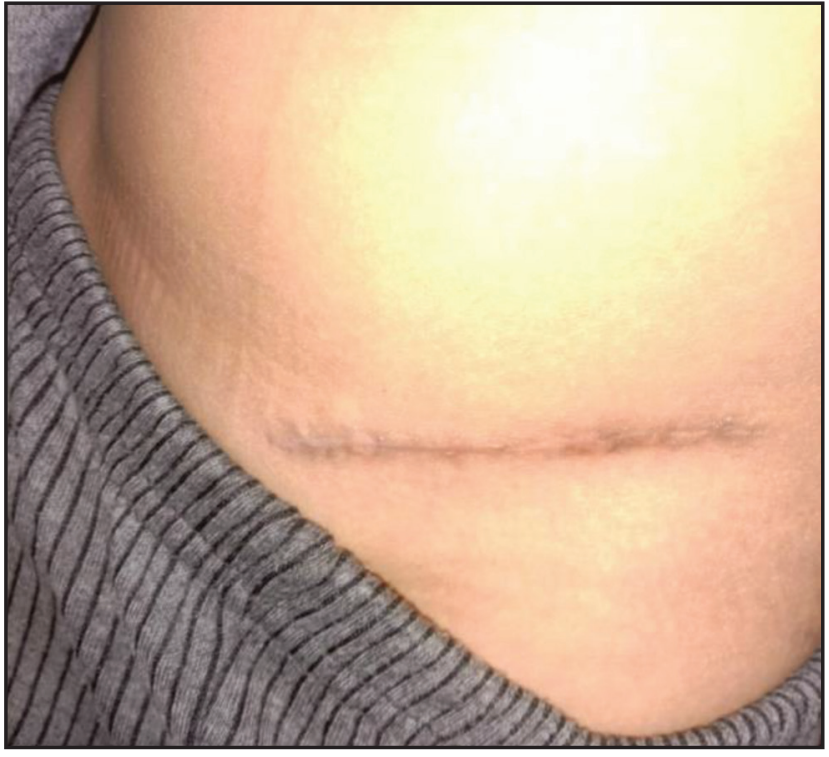

Fig. 9 Scar in another patient at 5-month postoperative follow-up.

\section{Conflict of Interest}

None.

\section{References}

1 Palkar VM. Full-thickness skin grafting. J Surg Oncol 2000;73(1):31

2 Osman OF, Emara S. Extended use of full-thickness skin grafts, employing variable donor sites. World J Plast Surg 2018;7(2):159-165

3 Kim S, Chung SW, Cha IH. Full thickness skin grafts from the groin: donor site morbidity and graft survival rate from 50 cases. J Korean Assoc Oral Maxillofac Surg 2013;39(1):21-26 